\title{
Furthering Media Independence Mechanisms for Future Internet Enablement
}

\author{
D. Corujo ${ }^{1}$, M. Lebre ${ }^{2}$, D. Gomes ${ }^{3}$, R. L. Aguiar ${ }^{4}$ \\ Instituto de Telecomunicações, Universidade de Aveiro \\ Aveiro, Portugal \\ $\left\{\right.$ dcorujo $^{1}$, marcelolebre ${ }^{2}$, dgomes ${ }^{3}$, ruilaa $\left.{ }^{4}\right\} @$ av.it.pt
}

\begin{abstract}
The utilization of Media Independent Handover (MIH) mechanisms, such as the ones provided by the IEEE802.21 standard, allow the abstraction of control and information querying primitives of different wireless access technologies, using a common interface. This not only simplifies the design of high-level entities interfacing with said technologies, but by providing a transport protocol for that interface, such primitives can be used to extend controlling mechanisms towards remote entities. However, the standard only employs such flexible and abstraction capabilities towards handover optimization and facilitation scenarios. In this paper, we take advantage of the flexible design of the MIH mechanisms, and propose evolutions over their base design, allowing their integration into the areas of Internet Multimedia Optimization and the Internet of Things.
\end{abstract}

Keywords-IEEE802.21; Video; Internet of Things; Sensors

\section{INTRODUCTION}

Today's widespread availability of different kinds of wireless technologies allows mobile users to access a plethora of services and rich multimedia content from the Internet. Moreover, it also enables them to generate and disseminate their own information, using the Internet as a vehicle for sharing different kinds of content in web-based social networks. In parallel, this on-line wireless interconnectivity has also motivated applications featuring data provided by sensor networks, whose generated data can be used to remotely trigger, optimize or detail different kinds of industrial, commercial and entertainment processes.

In these highly heterogeneous scenarios, it is truly complex to create and manage the necessary conditions for deploying such applications in a shared environment like the Internet: different wireless technologies, providing access to various services over distinct kinds of devices, each with its own set of diverse features, requirements, supported technologies and protocols. This complexity is furthermore aggravated when considering that mobile terminals can be on the move, or have different restrictions when it comes to processing capabilities and battery consumption.

Managing the deployment and execution of such scenarios under these conditions, requires controlling entities in the network to consider a plethora of different variables, which can cross different protocols, datasets and layers of the network stack. Furthermore, with the increase in number and kind of devices able to connect to the Internet, the amount of variables will only increase.

This work has been partially funded by the European Community's Seventh Framework Programme (FP7-ICT-2009-5) under grant agreement no. 258053 (MEDIEVAL project), and by the Portuguese Foundation for Science and Technology (FCT) grant agreement SFRH / BD / 61629 / 2009.
In this article, we analyze the Media Independent interfacing abstraction abilities of the recent IEEE802.21 standard [1], and exploit their flexibleness by enhancing them beyond the original concept of handover support, considering as well: i) support for multimedia-related events and commands for connectivity optimization in multimedia scenarios and ii) support for sensor-based abstraction, enabling Internet of Things (IoT) scenarios.

The remainder of this article is organized as follows. Section II presents related work on Future Internet solutions considering the separated use cases of multimedia traffic, IoT scenarios and media independent mechanisms. Section III and IV detail the evolutions done over the IEEE802.21 for supporting multimedia-enhanced handovers, and IoT scenarios, respectively. Finally, we conclude in Section V and highlight future work.

\section{RELATED WORK}

\section{A. Video Traffic Boom in the Future Internet}

A specific Internet architectural evolution area being addressed in recent research efforts, considers the expected huge increase of video as the predominant source of Internet traffic in the coming years [2]. The surge of mobile terminals able to connect to the Internet, as well as the availability of video content online, have motivated network solution providers to develop mechanisms supporting video services more efficiently. So far, only isolated evolutions have surfaced, with solutions focusing in video support enhancements for specific access technologies [3][4], transport protocols [5][6], application mechanisms [7][8] and broadcast/multicast techniques [9][10]. These isolated improvements only tackle specific problem spaces, and thus do not provide a solution that is able to consider different functional areas addressing an enhanced online video experience. In this respect, the MEDIEVAL [11] project (MultiMEDia transport for mobIlE Video AppLications) is developing an approach towards the efficient support of video services over wireless access networks with mobility support: it considers the deployment of a common framework integrating different standardization and research solutions for video optimization, using the IEEE802.21 standard as a linking point between the different functional entities. 


\section{B. IoT Environments}

The continuous miniaturization of electronics, along with the development of different Personal Area Networks wireless technologies (i.e., Bluetooth, ZigBee) have enabled the deployment of small networkable devices targeting the optimization of industrial, commercial and entertainment processes. Smart Environment scenarios thus become possible, featuring complex heterogeneous environments, composed by widely varied kinds of devices and technologies. To shine a guiding light into these different possibilities, different standardization initiatives led by the 3GPP [12] and ETSI [13], have started to establish the operator-driven fabric for supporting Machine-to-Machine (M2M) communications. These approaches are inspired by Service-Oriented Architecture (SOA) methodologies, which aim to bring a webservice based interfacing with sensors, actuators and underlying frameworks [14]. However, these methodologies often rely on different protocols to achieve discovery, management and other controlling procedures involving devices [15], and the webservice nature of some of the mechanisms places stringent requirements to devices that have low capacity and resources to support them. These considerations, often lead to the deployment of gateway-like designs, producing "islands" of solutions supporting specific technologies only.

\section{Media Independent Mechanisms}

The proliferation of mobile terminals equipped with multiple wireless interfaces, raised the need to support Always Best Connected scenarios [16] where the different access technologies could be exploited by the devices, services and network operators. In this sense, the Media Independent Handovers (MIH) IEEE802.21 standard provided a common framework to simplify and optimize handover procedures involving different access technologies.

MIH-enabled nodes are coupled with an abstracting MIH Function (MIHF) which acts as a translator between high-level entities (here dubbed MIH-Users) and link layer modules. MIH-Users interface with the MIHF using a MIH Service Access Point (SAP), which not only gives access to a set of Event, Command and Information services, but also maps such services into each technology-specific respective primitive (supporting 802.3, 802.11, 802.16 and 3GPP).

Through the provision of these facilities in a local and remote way, the flexible design of the framework allows for the definition of both mobile and network-controlled optimized mobility schemes in different kinds of environments [17]. Moreover, the IEEE802.21 framework also provides intrinsic service management mechanisms for MIH-enabled node discovery, registration, capability discovery, and event threshold configuration.

Recently, efforts have been taken to further explore these media independence concepts beyond their original technology set [18], but still under network handover application. We argue that the joint MIHF and MIH SAP, along with the remote availability of the Event, Command and Information services, can be further extended to support not only different link technologies, but also new applications beyond handover. In the following sections, we illustrate and discuss the necessary extensions to support multimedia optimizations and interfacing with IoT devices, such as sensors.

\section{SUPPORTING A MUltimedia INTERnET}

The MEDIEVAL Project aims to evolve the Internet architecture towards efficient video transport. It aims to enhance network-related processes (wireless access management, mobility and traffic optimization) with information about the characteristics of the video service being transported (such as video characteristics, number of layers, bit rate requirements), as well as providing network status information to video services [20].. This approach not only allows the network to adjust its procedures based on the characteristics of the video traffic, but also allows video services to configure themselves considering network conditions.

In addition, it follows a cross-layer design that exploits the interaction between the different layers of the network stack, achieving video performance values unattainable with individual developments. In order to integrate these procedures, a cross-layer abstraction approach becomes necessary. In this sense, the MEDIEVAL project enhances the information capabilities of the IEEE802.21 standard, by including video information in a broader range of abstract networking procedures.

\section{A. Media Independent Video Information}

The MEDIEVAL project is integrating video-specific information parameters, which can be interexchanged between different stack layer components for enhancing video processes. In one hand, new Information Elements from the IEEE802.21 Information Service have been defined (providing information such as the support of Multimedia Broadcast/Multicast Service or Content Delivery Networks), which enable video-aware handover candidate selection for mobile terminals.

Regarding Events and Commands, extensions to the mediaindependent signaling functionalities provided by IEEE802.21 to support specific mobility functionalities (e.g.: related to new mobility protocols such as Distributed Mobility Management) have been defined, supported by added information about link quality and QoS levels (which is useful for mobility management entity decisions). In addition, to support differentiated processing of video-marked packet flows, the IEEE802.21 signaling has been further enhanced with flow and packet marking awareness. This enables entities controlling such features to utilize the enhanced 802.21 protocol for those procedures. Table I provides a summary of presented extensions, which empower the IEEE802.21 signaling with new primitives and parameters assisting and optimizing link operations towards the support of video services in mobile environments.

TABLE I. $\quad 802.21$ PROTOCOL ENHANCEMENTS

\begin{tabular}{|c|l|}
\hline Parameter & \multicolumn{1}{c|}{ Change } \\
\hline Link Action Type & $\begin{array}{l}\text { New link actions have been developed with the } \\
\text { purpose of changing flow attributes at a link interface, } \\
\text { and to execute resource configurations for video }\end{array}$ \\
\hline
\end{tabular}




\begin{tabular}{|c|l|}
\hline Parameter & \multicolumn{1}{c|}{ Change } \\
\hline $\begin{array}{c}\text { Flow } \\
\text { Identification }\end{array}$ & $\begin{array}{l}\text { Identify flows used for video transport based on IP } \\
\text { destination/source, port destination/source and } \\
\text { protocol }\end{array}$ \\
\hline Flow Attributes & $\begin{array}{l}\text { The protocol enables entities to understand } \\
\text { parameters related with video flows and packet } \\
\text { marking characteristics such as drop eligibility, } \\
\text { Quality of Service and Multicast support. }\end{array}$ \\
\hline $\begin{array}{c}\text { New Link } \\
\text { Parameter } \\
\text { Events }\end{array}$ & $\begin{array}{l}\text { Provide information about system load, number of } \\
\text { users at a network point, congestion window and } \\
\text { transmission rate. }\end{array}$ \\
\hline \multicolumn{2}{|l}{} \\
\hline
\end{tabular}

\section{B. New Media Independent Networking Procedures}

With the increase of wireless users accessing video services online, it becomes complex even for MIH-aided mechanisms to exercise control over hundreds and thousand of individual mobile terminals. In this sense, we have defined base multicast signaling mechanism for the IEEE802.21 messages to reach not only single users, but also groups of users, as shown in Figure 1.

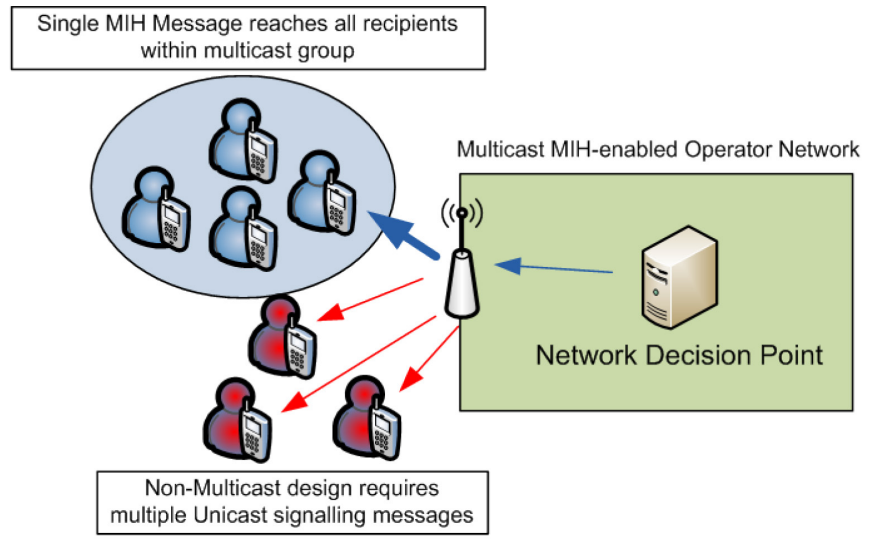

Figure 1 - MIH Multicast-Enhanced Mechanisms

By enhancing the per-node unique MIHF identifier with multicast group identification and registration capabilities, we are able to have network decision entities sending a single message for controlling several users that join a multicast group.

Parallel to this, the standard defines Information Servers which act as repositories of Information Elements, composing the IEEE802.21 information Service. These servers are queried by MIH-enabled mobile terminals, for obtaining information on the characteristics of available network Points of Attachment (PoA). In this way, the mobile terminal is able to execute an informed handover.

However, this querying process, when done to a single central Information Server that is responsible for a large number of access and domain networks, can produce delayed and lengthy query results. To optimize this process, the Information Service querying has been enhanced through a hierarchical process, where different functional network domains are governed by different interconnected information servers [19]. In this case, the local Information Server responds to queries about its local PoAs, allowing for brief query results. Whenever a mobile terminal queries the server about a PoA belonging to another domain, the Information Server is able to relay that query to a higher hierarchical located server. In turn, this server is able to forward this query to the specific Information Server responsible for that domain, and then forward the reply back to the original requester.

\section{New Access Technology Operations}

The base IEEE802.21 framework includes link-specific SAPs for Ethernet, WLAN, WiMAX, 3GPP and 3GPP2 link technologies. However, later developments of cellular technologies, such as 3GPP LTE-A, are not provided with a 802.21 primitive mapping in the standard. In this sense, the project is developing a specific LTE-A SAP (as shown in Figure 2), allowing the support and control of such interfaces in the MEDIEVAL framework.

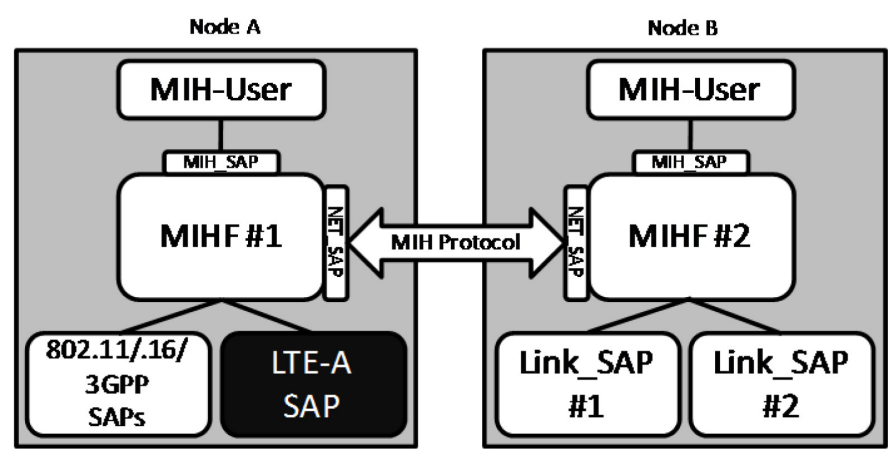

Figure 2 - New SAP interfacing with LTE-A links

Moreover, this extension allows the normal 802.21 to be coupled with parameters not defined in the base standard (such as Reference Signal Received Power and Reference Signal Received Quality), which can be used to further enhance video support at the wireless link layers [21]

\section{MEdia IndEPENDENT INTERNET OF THINGS}

A major challenge in addressing IoT scenarios is how to interface with the different existing devices, such as sensors. Different makers provide different interfaces and protocols for controlling and accessing the information obtained by sensors. To facilitate the deployment of different sensor technologies in IoT scenarios, we enhanced the original media independent design with sensor mechanisms, whose abstract functionality facilitates operations involving such devices. These extensions are presented in the following sections.

\section{A. Sensor SAP Design}

Our proposed enhancement involves the definition of a Sensor SAP for controlling and accessing information obtained by sensors, and providing it to upper layers in different entities. This SAP extends the existing set of 802.21 messages, with the sensor variants indicated in Table II. 
TABLE II. SENSOR SAP SERVICE SET

\begin{tabular}{|c|l|}
\hline Service & \multicolumn{1}{c|}{ Message } \\
\hline \multirow{4}{*}{ Management } & MIH_Sensor_Capability_Discover.request \\
& MIH_Sensor_Capability_Discover.response \\
& MIH_Event_Subscribe.request \\
& MIH_Event_Subscribe.response \\
& MIH_Event_Unsubscribe.request \\
& MIH_Event_Unsubscribe.response \\
\hline \multirow{4}{*}{ Commands } & MIH_Sensor_Configure_Thresholds.request \\
& MIH_Sensor_Configure_Thresholds.response \\
& MIH_Sensor_Action.request \\
& MIH_Sensor_Action.response \\
\hline \multirow{2}{*}{ Events } & MIH_Sensor_Event.indication \\
& MIH_Sensor_Parameter_Report.indication \\
\hline
\end{tabular}

These new primitives address services enabling: i) Management commands allowing sensor capability discovery and event subscription/unsubscription; ii) Commands related to the execution of sensor actions (e.g., activate or deactivate sensors, force sleep mode), as well as event threshold configuration, and iii) primitives used for sensor event dissemination. To complement these new messages, new supporting datatypes have also been defined, able to carry sensor-related information, as defined in Table III.

TABLE III. SENSOR SAP DATATYPES

\begin{tabular}{|c|c|}
\hline Datatype & Description \\
\hline Sensor Identifier & Sensor identification (Variable) \\
\hline Req. MIH Sensor Event List & $\begin{array}{l}\text { List of supported sensor events (32bit } \\
\text { Bitmap) }\end{array}$ \\
\hline Req. MIH Sensor Command List & $\begin{array}{l}\text { List of support sensor commands (32bit } \\
\text { Bitmap) }\end{array}$ \\
\hline Sensor Event Configuration List & $\begin{array}{l}\text { Configurations List for each sensor type } \\
\text { (Variable) }\end{array}$ \\
\hline Sensor Parameter Report List & $\begin{array}{l}\text { List of sensor parameters to report } \\
\text { (Variable) }\end{array}$ \\
\hline Sensor Identifier List & List of sensor identifiers (Variable) \\
\hline Sensor Device States Response & $\begin{array}{l}\text { Used to report the device status } \\
\text { (Variable) }\end{array}$ \\
\hline Sensor Configure Request List & $\begin{array}{l}\text { List of configuration parameters } \\
\text { (Variable) }\end{array}$ \\
\hline Sensor Configure Response List & List of configuration status (Variable) \\
\hline Sensor Action & $\begin{array}{l}\text { Definition of the required action to be } \\
\text { performed (Unsigned Integer ( } 8 \mathrm{bits}) \text { ) }\end{array}$ \\
\hline
\end{tabular}

This approach allowed for the abstraction of sensor details, while reutilizing the same media independent message structure, encoding rules and general frame format outlined by the 802.21 standard.

\section{B. Extensions to Sensor SAP and MIHF Interfacing}

Sensors are often low-powered devices with a stringent set of memory and processing capabilities. In order to reduce the requirements imposed on sensors by our media independent sensor enhancements, we extended the original framework by allowing nodes to be equipped with only the Sensor SAPs, and connecting to a MIHF-enabled gateway node, able to execute any MIHF related operation on their behalf (thus freeing the node of such responsibilities and processing demands).

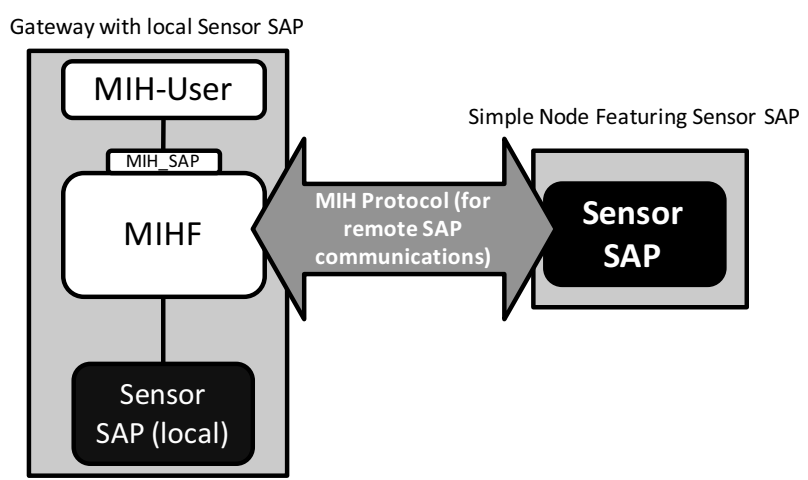

Figure 3 - Remote SAP Access for Low-powered Devices

This approach (shown in Figure 3) not only allows the exploitation of remote interactions between the MIHF and SAPs, but also opens the way for exploring sensor networking technologies, such as 802.15.4 (enhancing the set supported wireless technologies for 802.21 signaling transport). Moreover, it allows the usage of MIH mechanisms even in low-powered devices, which is often the case in IoT scenarios featuring sensor devices.

\section{CONCLUSION AND FUTURE WORK}

The technological evolutions in Information and Communication Technologies, not only in terms of exciting new services, but also in the underlying background technological breakthroughs that support them, have forever changed human interaction with the digital realm (and more importantly, what we expect from this digital realm). Today, information widespread is a communication vehicle that can be used for entertainment purposes (i.e., social networking) and for commercial and industrial purposes (i.e., tracking components, monitoring processes). By associating this information pervasiveness to wireless dissemination mechanisms, the interaction scenarios (not only between man and machine, but also between machines), the amount of prospect scenarios increases to almost limitless possibilities.

However, the resulting overabundance of information increasingly stresses the underlying fabric of the Internet architecture, which is motivating the adoption of new supportive mechanisms. To deal with the heterogeneity imposed in wildly varied environments, it becomes important to provide a common framework which can be used by different processes for interfacing with the different functional areas (and entities) involved.

In this paper, we first introduced the IEEE802.21 Media Independent Handover services standard, highlighting the flexible nature of its cross-layer design through the usage of SAPs, and remote transport protocol of its different provided services. Then, we leveraged this flexible design and presented a set of core changes to the standard that extended its set of supported procedures. By extending the encompassed events, 
commands and information services by adding new SAP and parameter support, we were able to re-utilize MIH mechanisms in scenarios beyond the original use case of handover. These scenarios, allow the utilization of these mechanisms into key areas of Internet architectural evolution, such as Multimedia Networking and the Internet of the Future.

Some of these mechanisms have already been demonstrated, through the actual implementation of the enhanced procedures over the flexible MIH framework. Taking as base the ODTONE 802.21 implementation [22], the MIH control of sensor devices in IoT scenarios has been successfully deployed and even enabled novel scenarios, such as energyefficient wireless interface control [23] and information dissemination [24]. However, we argue that this enhancement does not need to stop here. Currently, we are furthering these concepts even more, by defining the means for MIHFs to detect, select and automatically learn how to interface with different entities, through dynamic SAP allocation. The objective is to provide a true media independent control layer, able to interact with a plethora of different devices and services, without having to be tied to pre-knowledge of interfacing procedures.

\section{REFERENCES}

[1] IEEE 802.21 Standard, "Local and Metropolitan Area Networks - Part 21: Media Independent Handover Services", January 2009

[2] Cisco Visual Networking Index: Global Mobile Data Traffic Forecast Update, Cisco White Paper [Online]. Available: http://www.cisco.com/en/US/solutions/collateral/ns341/ns525/ns537/ns7 05/ns827/white_paper_c11-520862.pdf

[3] IEEE P802.11aaTM D3.01 Draft Standard for Information TechnologyTelecommunications and information exchange between systems- Local and metropolitan area networks- Specific requirements- Part 11: Wireless LAN Medium Access Control (MAC) and Physical Layer (PHY) specifications, Amendment 3: MAC Enhancements for Robust Audio Video Streaming, 2011

[4] Munaretto, D.; Jurca, D.; Widmer, J.; , "Broadcast video streaming in cellular networks: An adaptation framework for channel, video and ALFEC rates allocation," Wireless Internet Conference (WICON), 2010 The 5th Annual ICST, vol., no., pp.1-9, 1-3 March 2010

[5] Wenger, S.; Ye-Kui Wang; Schierl, T.; , "Transport and Signaling of SVC in IP Networks," Circuits and Systems for Video Technology, IEEE Transactions on, vol.17, no.9, pp.1164-1173, Sept. 2007

[6] A. Vitali, A. Borneo, M. Fumagalli and R. Rinaldo "Video over IP using standard-compatible multiple-description coding: An IETF Proposal", J. Zhejiang Univ. SCIENCE, vol. 7, pp.668 2006

[7] Li Yan; Gao Yang; , "A Web Video Service Based on Flash Video Technique," Internet Technology and Applications (iTAP), 2011 International Conference on , vol., no., pp.1-4, 16-18 Aug. 2011

[8] Vidal, I.; Garcia-Reinoso, J.; Valera, F.; Bikfalvi, A.; , "Enabling layered video coding for IMS-based IPTV home services," Network, IEEE , vol.23, no.6, pp.30-35, November-December 2009
[9] J. Antoniou, C. Riede, F.C. Pinto, A. Pitsillides, "Context-Aware Multiparty Session Support for Adaptive Multicasting in Heterogeneous Mobile Networks" 2nd International ICST Conference on Mobile, Wireless Middleware, Operating Systems and Applications (Mobilware 2009), Dresden, Germany, April 2009

[10] Gruber, M.; Zeller, D.; , "Multimedia broadcast multicast service: new transmission schemes and related challenges," Communications Magazine, IEEE , vol.49, no.12, pp.176-181, December 2011

[11] FP7 EU project: MultimEDia transport for mobIlE Video AppLications (MEDIEVAL), Grant agreement no. 258053.

[12] 3GPP TR 22.868 V8.0.0 (2007-03), Study on Facilitating Machine to Machine Communication in 3GPP Systems (Release 8)

[13] ETSI TS 102689 V1.1.1 (2010-08), Technical Specification, Machineto-Machine communications (M2M); M2M service requirements

[14] C. Thiede, C. Tominski, H. Schumann, Service-oriented information visualization for smart environments, in: Proceedings of the $200913^{\text {th }}$ International Conference Information Visualisation, IEEE Computer Society, Washington, DC, USA, 2009, pp. 227-234.

[15] Web Services Platform Architecture : SOAP, WSDL, WS-Policy, WSAddressing, WS-BPEL, WS-Reliable Messaging, and More, Prentice Hall PTR, 2005.

[16] Gustafsson, E.; Jonsson, A.; , "Always best connected," Wireless Communications, IEEE, vol.10, no.1, pp. 49- 55, Feb. 2003

[17] Telemaco Melia, Antonio de la Oliva, Albert Vidal, Ignacio Soto, Daniel Corujo, and Rui Aguiar. Toward IP converged heterogeneous mobility: A network controlled approach. Computer Networks, 51(17), 2007.

[18] Antonio de la Oliva, Ignacio Soto, Albert Banchs, Johannes Lessmann, Christian Niephaus, and Telemaco Melia. IEEE 802.21: Media independence beyond handover. Computer Standards \& Interfaces, 33(6):556-564, 2011.

[19] Daniel Corujo, Fabio Buiati, Luis Villalba, João Soares, Susana Sargento, Rui L. Aguiar, "Hierarchical Neighbor Discovery Scheme for Handover Optimization"IEEE Communication Letters, ISSN 1089-7798, Sep 2010

[20] Daniel Corujo, Albert Banchs, Telemaco Melia, Michelle Wetterwald, Leonardo Badia, Rui L. Aguiar, "Video-Enhancing Functional Architecture for the MEDIEVAL Project", Chapter in Mobile Networks and Management, Springer Berlin Heidelberg, ISBN: 978-3-642-214448, Jan 2011

[21] Mezzavilla, M.; Wetterwald, M.; Badia, L.; Corujo, D.; de la Oliva, A.; , "Wireless access mechanisms and architecture definition in the MEDIEVAL project," Computers and Communications (ISCC), 2011 IEEE Symposium on, vol., no., pp.31-36, June 28 2011-July 12011

[22] Corujo, D.; Guimaraes, C.; Santos, B.; Aguiar, R.L.; , "Using an opensource IEEE 802.21 implementation for network-based localized mobility management," Communications Magazine, IEEE , vol.49, no.9, pp.114-123, September 2011

[23] Daniel Corujo, Marcelo Lebre, Diogo Gomes, Rui L. Aguiar, "Sensor Context Information for Energy-Efficient Optimization of Wireless Procedures ", Proc. 22nd IEEE International Symposium on Personal, Indoor and Mobile Radio Communications (PIMRC), Toronto, Canada, Sep 2011

[24] Daniel Corujo, Marcelo Lebre, Diogo Gomes, Rui L. Aguiar, "A Framework for Flexible Sensor Information Dissemination", Proc. 2nd International Workshop on Interconnections of Wireless Sensor Networks, Barcelona, Spain, Jun 2011 